\title{
Preservation of functional hearing after gamma knife surgery for vestibular schwannoma
}

\author{
Zachary N. Litvack, M.D., Georg Norén, M.D., Ph.D., Prakash B. Chougule, M.D., \\ AND Zhen Zheng, PH.D.
}

New England Gamma Knife Center and Departments of Clinical Neurosciences (Neurosurgery), Radiation Oncology, and Medical Physics, Brown Medical School /Rhode Island Hospital, Providence, Rhode Island

\begin{abstract}
Object. The goal of this retrospective study was to define the rates of preservation of functional hearing and growth control of vestibular schwannomas (VS) treated by gamma knife surgery (GKS) involving a consistent 12-Gy prescription dose.

Methods. One hundred thirty-four patients with unilateral VS underwent GKS between 1994 and 2000. The mean magnetic resonance (MR) imaging follow-up period was 31.7 months (maximum 72 months), and the mean audiometry follow-up interval was 26.3 months (maximum 60 months). The mean marginal dose was $12 \pm 0.6 \mathrm{~Gy}$. The mean maximum dose delivered to the tumor center was 25.4 Gy (range 17.4-34.3 Gy). The tumor control rate, defined as no change or a reduction in size at last follow up, was $96.7 \%$. Of the patients studied, $97.7 \%$ remained free from the need to undergo tumor resection. Overall functional hearing preservation was $61.7 \%$; the preservation rate for intracanalicular tumors was $63.6 \%$, for those with an intracranial diameter less than $1.5 \mathrm{~cm}$ it was $54.5 \%$, for those between 1.5 and $3 \mathrm{~cm}$ it was $68.2 \%$, and for those larger than $3 \mathrm{~cm}$ it was $33.3 \%$. Early in the series, three patients $(2.2 \%)$ developed temporary facial weakness (House-Brackmann Grade II-III) in the posttreatment period, but this resolved within a few weeks. No case of facial weakness occurred after 1996.

Conclusions. The authors demonstrated the efficacy, safety, and in many ways, the advantage of GKS over microsurgery for VS. Patients harboring tumors $3 \mathrm{~cm}$ or smaller in intracranial diameter, regardless of their age and medical condition, should be given the option of undergoing GKS as primary treatment.
\end{abstract}

\section{KEY WORDS • vestibular schwannoma • acoustic neuroma • gamma knife • radiosurgery - hearing preservation}

In recent years, preservation of hearing has become an increasingly important issue in the management of VS. Because complete removal of the lesion and preservation of facial nerve function are routinely expected at centers of excellence, the next important achievement is preservation of functional hearing. In selected series in which small tumors have been resected by experienced surgeons, the authors have demonstrated that hearing, when functional preoperatively, can be preserved in 40 to $70 \%$ of the cases. ${ }^{12,35,36}$ Preservation of functional hearing after excision of medium-sized and large tumors, however, is technically demanding, and the preservation rate is less impressive than that for small lesions. $4,6,11,12,15,19,22,23,28,29,35-37$ In several studies of GKS for VS investigators have published more promising results. ${ }^{7,10,16-18,20,24-27,30}$

This retrospective study was undertaken to study the potential of functional hearing preservation and growth control in VS when GKS is administered in a consistent manner with regard to dose level and dosimetry.

Abbreviations used in this paper: GKS = gamma knife surgery; LINAC = linear accelerator; $\mathrm{MR}=$ magnetic resonance; $\mathrm{SRT}=$ stereotactic radiotherapy; VS = vestibular schwannoma.

\section{CLINICAL MATERIAL AND METHODS}

All patients in whom the senior author (G.N.) performed GKS at the New England Gamma Knife Center between October 1994 and February 2000 were identified as the study cohort for analysis. Data were collected from all medical records. There were 134 patients with unilateral VS. Of these patients, 70 were female and 64 male, who ranged in an age from 13 to 86 years (mean age 55.3 years) at the time of treatment. Sixty-two tumors were right sided and 72 left sided. Because one patient required repeated GKS for further progression of his tumor, a total of 135 treatments were performed (Table 1).

Eighteen patients underwent subtotal tumor resection prior to GKS. In nine of these, GKS was the second part of a planned comprehensive treatment. In five patients, an intended complete excision was not achieved because of profuse hemorrhage, tumor tissue attached to the facial nerve, or other technical reasons. In four patients recurrences unexpectedly developed after seemingly complete resection. Four patients underwent GKS for a second time after it failed to effect a cure prior to this study period. A summary of the pretreatment characteristics is found in Table 1. 
TABLE 1

Characteristics of patients with VS prior to GKS

\begin{tabular}{lc}
\hline \hline \multicolumn{1}{c}{ Characteristic } & No. of Cases $(\%)$ \\
\hline no. of patients & 134 \\
age (yrs) & \\
$\quad$ mean & 55.3 \\
$\quad$ range & $13-86$ \\
female & $70(52)$ \\
male & $64(48)$ \\
prior GKS & $4(3)$ \\
prior microsurgery & $18(13)$ \\
subjective hearing loss & $123(92)$ \\
tinnitus & $72(54)$ \\
dizziness & $60(45)$ \\
facial numbness & $31(23)$ \\
facial spasm & $5(4)$ \\
\hline
\end{tabular}

Facial nerve function was graded using the HouseBrackmann grading system. ${ }^{14}$ Pre-GKS and post-GKS audiological evaluation included tone and speech audiometry from which a pure tone average-based on three frequencies $(0.5,1,2 \mathrm{kHz})$-and speech discrimination scores were obtained. The results were graded according to the Gardner-Robertson classification. ${ }^{11}$

To enable an accurate and consistent comparison of findings on MR images, tumor size was defined by calculating the mean intracranial diameter according to measurements along three easily defined axes on Gd-enhanced $\mathrm{T}_{1}$-weighted images. The maximum anteroposterior and mediolateral distances were measured on axial images, parallel and perpendicular to the sagittal plane. The maximum craniocaudal diameter was determined by assessing coronal images. The mean diameter as well as the greatest intracranial dimension were noted for each tumor. A decrease or increase of size was regarded as significant if the change of mean diameter was at least $2 \mathrm{~mm}$. This cutoff was chosen based on limitations in spatial resolution of the standard 1.5-tesla MR imaging used for most follow-up studies. Intracanalicular tumors without intracranial extension were measured in all three dimensions, with change of size being defined as significant if the transverse diameter had decreased or increased $2 \mathrm{~mm}$ or more.

The GKS procedure included application of a Leksell stereotactic frame and evaluation with Gd-enhanced MR imaging. We acquired $\mathrm{T}_{1}-$ and $\mathrm{T}_{2}$-weighted sequences as well as two- and three-dimensional volume sequences, the latter initially with a $1.5-\mathrm{mm}$ slice thickness and later reduced to $1 \mathrm{~mm}$. The images were transferred to the Leksell GammaPlan workstation for careful analysis. The margins of the tumor and the locations of the trigeminal, facial, and cochlear nerves were traced. Dose plans were created with a very tight fit between the tumor periphery and in general the $50 \%$ isodose line in the direction of the cranial nerves (mean prescription isodose line $47.2 \pm$ $6 \%$ ). A mean prescription dose of $12 \mathrm{~Gy}( \pm 0.6 \mathrm{~Gy})$ was delivered to this isodose line. The mean maximum dose delivered to the tumor center was 25.4 Gy (range 17.434.3 Gy). The median number of isocenters (shots) per treatment was 15 (range 1-42). All treatments in this study were performed with a $201{ }^{60} \mathrm{Co}$ source gamma knife model U (Elekta Inc., Norcross, GA).
Follow-up study consisted of MR imaging and audiometry evaluations performed at 6 months, 1 year, annually for the first 5 years, and every 2 or 3 years thereafter.

\section{RESULTS}

\section{Hearing Preservation}

Hearing graded as Gardner-Robertson Class I or II frequently is defined as "useful" or "functional." Of the 134 patients in whom pretreatment audiograms were acquired, $53(39.5 \%)$ presented with Gardner-Robertson Class I or II hearing. Audiological follow-up studies were complete in $73.1 \%$ (98 patients) within a mean follow-up period of 26.3 months (range 12-60 months). Hearing was "functional" in 47 of these patients before radiosurgery and remained so post-GKS in $61.7 \%$ (29 patients) in a mean follow-up period of 26.3 months (range 12-50 months). In cases of intracanalicular tumors, functional hearing was preserved in seven $(63.6 \%)$ of 11 patients. In cases involving tumors between 1.5 and $3 \mathrm{~cm}$, hearing remained functional in $15(68.2 \%)$ of 22 patients (Table 2). The MR images in Fig. 1 demonstrate a $2.3-\mathrm{cm}$ tumor in a patient with Gardner-Robertson Class I hearing pre- and postGKS. In addition, hearing improved from nonfunctional to functional in three $(5.9 \%)$ of 51 patients after radiosurgery. Of these, two patients harbored tumors between 1.5 and $3 \mathrm{~cm}$ in maximum intracranial diameter, whereas the third tumor was smaller than $1.5 \mathrm{~cm}$.

\section{Tumor Control Rates}

The goal of the treatment of VS with stereotactic radiosurgery is defined as "growth control" or "lack of growth;" tumors that decrease in size and those that remain stable in size following radiosurgery fall within this category. In approximately $90 \%$ of patients (121 of 134) complete MR imaging studies were collected during a mean follow-up period of 31.7 months (range 12-72 months). In this group, the tumors in $56(46.3 \%)$ patients decreased in size, those in $61(50.4 \%)$ remained unchanged, and those in four (3.3\%) increased in size (Table $3)$. Of the tumors that grew after surgery, one was fewer than $1.5 \mathrm{~cm}$ and three were between 1.5 and $3 \mathrm{~cm}$ before treatment.

Another definition of success often used when reviewing radiosurgical results is freedom from the need for resection. Because of the lesion's continued growth, three patients required post-GKS resection, representing 2.2\% of the study population. These resections were performed at 13,26 , and 40 months after radiosurgery. In three pa-

TABLE 2

Results for preservation of hearing stratified by tumor size

\begin{tabular}{lccc}
\hline \hline & \multicolumn{2}{c}{ Patients W/ Functional Hearing } & \\
\cline { 2 - 3 } Tumor Size $(\mathrm{cm})$ & Preop & Postop & \% Preservation \\
\hline intracanalicular & 11 & 7 & 63.6 \\
$\leq 1.5$ & 11 & 6 & 54.5 \\
$1.5-3$ & 22 & 15 & 68.2 \\
$>3$ & 3 & 1 & 33.3 \\
\hline
\end{tabular}




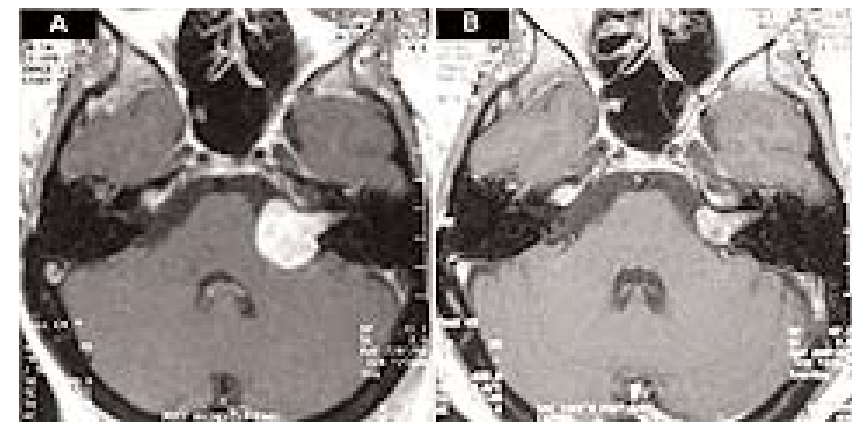

Fig. 1. Gadolinium-enhanced axial MR images obtained at treatment (A) and four years after GKS (B) demonstrating excellent growth control of a $2.3-\mathrm{cm}$ tumor in a patient in whom Gardner-Robertson Class I hearing was retained.

tients a shunt had been placed prior to GKS. A ventricular shunt for hydrocephalus was required in four patients after treatment. In these patients the mean intracranial tumor diameter was 1.6 to $3.3 \mathrm{~cm}$ prior to and 1.3 to $3.2 \mathrm{~cm}$ after treatment.

\section{Rates of Facial Nerve Dysfunction}

Facial weakness was present in 10 patients prior to GKS; five after subtotal and four after complete tumor resection. The tenth, an 81-year-old patient, developed weakness (House-Brackmann Grade III) after failure of a 3.5 $\mathrm{cm}$ partially cystic VS to respond to GKS and was retreated. Five of the 10 patients suffered complete facial paralysis (House-Brackmann Grade VI). Consequently, of the population studied, 129 (96.3\%) of 134 patients were at risk of developing new or increased facial weakness. Of those at risk, impairment of facial nerve function occurred in three $(2.3 \%)$ after radiosurgery. Facial nerve function in these patients was House-Brackmann Grade I prior to radiosurgery, which declined transiently for a few weeks' duration to Grades II to III and resolved completely thereafter. No case of facial weakness of any degree after GKS has occurred since 1996.

Prior to GKS, five $(3.7 \%)$ of 134 patients presented with hemifacial spasm. In one of these five GKS had been conducted prior to inclusion into this study. None of these five had previously undergone microsurgery. The spasm resolved in four patients during the posttreatment period. Eight $(6.2 \%)$ of 129 patients at risk developed new temporary hemifacial spasm in the post-GKS period.

\section{Rates of Trigeminal Nerve Dysfunction}

Twenty-three percent (31) of the patients suffered some degree of facial numbness before GKS. Of the 31 patients with preexisting numbness, nine (29\%) experienced complete resolution after GKS. In none of these patients did the facial hypesthesias worsen after treatment. Of the 103 patients with normal facial sensation prior to treatment, de novo transient hypesthesias developed in six patients $(5.8 \%)$ following the treatment.

\section{DISCUSSION}

Many clinical studies have shown that preservation of
TABLE 3

Results for growth control, stratified by pretreatment tumor size

\begin{tabular}{lccc}
\hline \hline & \multicolumn{3}{c}{ Posttreatment Growth, No. of Cases (\%) } \\
\cline { 2 - 4 } Tumor Size $(\mathrm{cm})$ & Decreased & No Change & Increased \\
\hline intracanalicular & 3 & 20 & 0 \\
$\leq 1.5$ & 10 & 20 & 1 \\
$1.5-3$ & 36 & 19 & 3 \\
$>3$ & 7 & 2 & 0 \\
total & $56(46.3)$ & $61(50.4)$ & $4(3.3)$ \\
\hline
\end{tabular}

useful hearing is more difficult to achieve than complete resection or growth control and preserved facial nerve function in the treatment of VS, regardless of treatment modality. This is especially true in the microsurgical series, in which the cochlear system is often sacrificed to undertake a complete resection (Table 4). Norén, et al., ${ }^{27}$ reviewed 254 GKS-treated tumors at the Karolinska Institute between 1969 and 1991, of which 132 tumors were associated with a pure tone accuity of better than 90 $\mathrm{dB}$; they reported hearing preservation in $77 \%$ of patients. In a review of 71 patients treated at the same institution between 1989 and 1990 , Norén ${ }^{25}$ showed a hearing preservation rate of $75 \%$ (12 of 16 cases) in patients with Gardner-Robertson Grade I/II at 1 year and 60\% at 2 years. It was concluded that any impairment of hearing in general occurs within 1 year from GKS and usually remains stable thereafter. ${ }^{13}$ Investigators at the University of Pittsburgh reported preservation of functional hearing, as defined previously, to be between 35.1 and $71 \%$ in different series treated at their institution..$^{7-9,18,20,30}$ Functional hearing preservation of 50\% was achieved in cases of small tumors with a pons-petrous diameter of less than 10 $\mathrm{mm} .{ }^{29}$ Foote, et al. ${ }^{10}$ reported a functional hearing preservation rate of $41.7 \%$.

Of recent reports that have been focused on preservation of hearing by administering reduced radiation doses, Prasad, et al., ${ }^{33}$ reported a useful hearing preservation rate of $40 \%$ when using a marginal dose of 13.3 Gy in a series of 153 patients. Flickinger, et al., ${ }^{7}$ reported a preservation

TABLE 4

Summary of hearing preservation rates after microsurgery for $V S^{*}$

\begin{tabular}{lc}
\hline \hline \multicolumn{1}{c}{ Authors \& Year } & Overall Hearing Preservation $\%^{\dagger}$ \\
\hline Nadol, et al., 1987 & 73 (IC only)/22 \\
Baldwin, et al., 1990 & 31.8 \\
Ebersold, et al., 1992 & 23.5 \\
Fischer, et al., 1992 & 29.3 \\
House \& Shelton, 1992 & $31-59$ \\
Nedzelski, et al., 1994 & 38 \\
Koos, et al., 1995 & 78 \\
Post, et al., 1995 & 39 \\
Samii \& Matthies, 1995 & 38 \\
Gormley, et al., 1997 & 38 \\
Rowed \& Nedzelski, 1997 & 50 (IC only)/29 \\
Samii \& Matthies, 1997 & 47 \\
\hline
\end{tabular}

* IC = intracanalicular.

$\dagger$ Authors vary in their definition of functional or useful hearing. 
rate of $71 \%$ when using a 13 -Gy dose in 190 patients. Petit, et al., ${ }^{31}$ demonstrated a preservation rate of $87 \%$ when administering $12 \mathrm{~Gy}$ in 45 cases. Collective findings in this sequence of reports suggests a direct relationship between hearing preservation and the marginal radiation dose. Furthermore, Petit, et al., interpreted this data to indicate that a steep portion of the dose-response curverelating to complications with cochlear nerve function as a function of marginal dose-had been reached and that further slight dose reduction may improve the function of that nerve. Andrews, et al., ${ }^{2}$ however, reported a 33\% preservation rate when applying a 12-Gy dose in 69 patients. With our functional hearing preservation rate of $61.7 \%$ when using 12 Gy in 98 patients-a rate that falls between those of the two previous studies ${ }^{2,31}$ - the exact slope of this portion of the dose-response curve has yet to be determined.

There are limited published data available regarding hearing preservation after single-dose LINAC-based radiosurgery for VS. The series from the University of Florida at Gainesville contained a majority of patients without useful hearing and thus pre- and posttreatment audiometry studies were not routinely obtained. ${ }^{21}$ Spiegelmann, et al. ${ }^{38}$ reported on 44 patients who underwent single-dose LINAC radiosurgery with an actuarial preservation rate of $71 \%$ but a $24 \%$ rate of facial weakness. These results are tempered by those of Suh, et al. ${ }^{39}$ who reported a hearing preservation rate of $29 \%$ after single-dose LINAC radiosurgery (prescription dose 8-24 Gy) for VS.

There is currently more interest in fractionated LINACbased SRT, resulting in an increase in published data for this modality. Andrews, et al., ${ }^{1}$ originally published results after fractionated SRT in seven patients with serviceable hearing. Immediately posttreatment, five (71.4\%) of seven patients still had serviceable hearing. In a more recent review by the same group the authors reported an $81 \%$ actuarial preservation rate at a median follow up of 38 weeks in a larger cohort of 56 patients. ${ }^{2}$ The results for fractionated SRT are promising but suffer from a lack of long term follow-up outcome data compared with GKS.

\section{CONCLUSIONS}

As microsurgical techniques for VS treatment have advanced over the decades, supported by better facial and cochlear nerve monitoring techniques, so has radiosurgery progressed with improved imaging and dose-planning techniques. With equivalent rates of tumor control across all modalities discussed, minimizing cranial nerve comorbidities has become the goal in establishing the primary treatment modality. This is now especially important with regard to hearing preservation.

Our overall hearing preservation rate of $61.7 \%$ in tumors up to $3.8 \mathrm{~cm}$ in size is comparable with or better than those reported in recent radiosurgical series. Gamma knife surgery is clearly superior to single-dose LINAC-based radiosurgery. Fractionated SRT remains promising but has yet to prove itself in the long term. Although microsurgery has yielded better results for intracanalicular and small tumors, the rate of functional hearing preservation demonstrated in the present study is superior to those reported in any microsurgical series for tumors greater than $1.5 \mathrm{~cm}$ in size. Given the high level of functional hearing preser- vation and growth control, we believe that GKS should be considered to be a primary treatment modality in any patient harboring a VS of up to $3 \mathrm{~cm}$ in intracranial diameter.

\section{References}

1. Andrews DW, Silverman CL, Glass J, et al: Preservation of cranial nerve function after treatment of acoustic neurinomas with fractionated stereotactic radiotherapy. Preliminary observations in 26 patients. Stereotact Funct Neurosurg 64: $165-182,1995$

2. Andrews DW, Suarez O, Goldman HW, et al: Stereotactic radiosurgery and fractionated stereotactic radiotherapy for the treatment of acoustic schwannomas: comparative observations of 125 patients treated at one institution. Int J Radiat Oncol Biol Phys 50:1265-1278, 2001

3. Baldwin DL, King TT, Morrison AW: Hearing conservation in acoustic neuroma surgery via the posterior fossa. J Laryngol Otol 104:463-467, 1990

4. DiTullio MV Jr, Malkasian D, Rand RW: A critical comparison of neurosurgical and otolaryngological approaches to acoustic neuromas. J Neurosurg 48:1-12, 1978

5. Ebersold MJ, Harner SG, Beatty CW, et al: Current results of the retrosigmoid approach to acoustic neurinoma. J Neurosurg 76:901-909, 1992

6. Fischer G, Fischer C, Remond J: Hearing preservation in acoustic neurinoma surgery. J Neurosurg 76:910-917, 1992

7. Flickinger JC, Kondziolka D, Niranjan A, et al: Results of acoustic neuroma radiosurgery: an analysis of 5 years' experience using current methods. J Neurosurg 94:1-6, 2001

8. Flickinger JC, Lunsford LD, Coffey RJ, et al: Radiosurgery of acoustic neurinomas. Cancer 67:345-353, 1991

9. Flickinger JC, Lunsford LD, Linskey ME, et al: Gamma knife radiosurgery for acoustic tumors: multivariate analysis of four year results. Radiother Oncol 27:91-98, 1993

10. Foote RL, Coffey RJ, Swanson JW, et al: Stereotactic radiosurgery using the gamma knife for acoustic neuromas. Int J Radiat Oncol Biol Phys 32:1153-1160, 1995

11. Gardner G, Robertson JH: Hearing preservation in unilateral acoustic neuroma surgery. Ann Otol Rhinol Laryngol 97: 55-66, 1988

12. Gormley WB, Sekhar LN, Wright DC, et al: Acoustic neuromas: results of current surgical management. Neurosurgery 41:50-60, 1997

13. Hirsch A, Noren G, Anderson H: Audiologic findings after stereotactic radiosurgery in nine cases of acoustic neurinomas. Acta Otolaryngol 88:155-160, 1979

14. House JW, Brackmann DE: Facial nerve grading system. Otolaryngol Head Neck Surg 93:146-147, 1985

15. House WF, Shelton C: Middle fossa approach for acoustic tumor removal. Otolaryngol Clin North Am 25:347-359, 1992

16. Kondziolka D, Lunsford LD: Preservation of hearing in acoustic neurinoma surgery. J Neurosurg 78:154-156, 1993

17. Kondziolka D, Lunsford LD, Flickinger JC: Acoustic neuromas. Curr Treat Options Neurol 4:157-165, 2002

18. Kondziolka D, Lunsford LD, McLaughlin MR, et al: Long-term outcomes after radiosurgery for acoustic neuromas. N Engl J Med 339:1426-1433, 1998

19. Koos WT, Matula C, Levy D, et al: Microsurgery versus radiosurgery in the treatment of small acoustic neurinomas. Acta Neurochir Suppl 63:73-80, 1995

20. Linskey ME, Lunsford LD, Flickinger JC, et al: Stereotactic radiosurgery for acoustic tumors. Neurosurg Clin N Am 3: 191-205, 1992

21. Mendenhall WM, Friedman WA, Buatti JM, et al: Preliminary results of linear accelerator radiosurgery for acoustic schwannomas. J Neurosurg 85:1013-1019, 1996 
22. Nadol JB Jr, Levine R, Ojemann RG, et al: Preservation of hearing in surgical removal of acoustic neuromas of the internal auditory canal and cerebellar pontine angle. Laryngoscope 97: 1287-1294, 1987

23. Nedzelski JM, Chiong CM, Cashman MZ, et al: Hearing preservation in acoustic neuroma surgery: value of monitoring cochlear nerve action potentials. Otolaryngol Head Neck Surg 111:703-709, 1994

24. Noren G: The Gamma Knife and acoustic tumors. Med Health R I 79:232-235, 1996

25. Noren G: Gamma knife radiosurgery for acoustic neurinomas, in Gildenberg PL, Tasker RR (eds): Textbook of Stereotactic and Functional Neurosurgery. New York: McGraw-Hill, 1998, pp 835-844

26. Noren G: Long-term complications following gamma knife radiosurgery of vestibular schwannomas. Stereotact Funct Neurosurg 70 (Suppl 1):65-73, 1998

27. Noren G, Greitz D, Hirsch A, et al: Gamma knife surgery in acoustic tumors. Acta Neurochir Suppl 58:104-107, 1993

28. Ogunrinde OK, Lunsford DL, Kondziolka DS, et al: Cranial nerve preservation after stereotactic radiosurgery of intracanalicular acoustic tumors. Stereotact Funct Neurosurg 64 (Suppl 1):87-97, 1995

29. Ogunrinde OK, Lunsford LD, Flickinger JC, et al: Cranial nerve preservation after stereotactic radiosurgery for small acoustic tumors. Arch Neurol 52:73-79, 1995

30. Ogunrinde OK, Lunsford LD, Flickinger JC, et al: Stereotactic radiosurgery for acoustic nerve tumors in patients with useful preoperative hearing: results at 2-year follow-up examination. J Neurosurg 80:1011-1017, 1994

31. Petit JH, Hudes RS, Chen TT, et al: Reduced-dose radiosurgery for vestibular schwannomas. Neurosurgery 49:1299-1307, 2001

32. Post KD, Eisenberg MB, Catalano PJ: Hearing preservation in vestibular schwannoma surgery: what factors influence outcome? J Neurosurg 83:191-196, 1995

33. Prasad D, Steiner M, Steiner L: Gamma surgery for vestibular schwannoma. J Neurosurg 92:745-759, 2000

34. Rowed DW, Nedzelski JM: Hearing preservation in the removal of intracanalicular acoustic neuromas via the retrosigmoid approach. J Neurosurg 86:456-461, 1997

35. Samii M, Matthies C: Hearing preservation in acoustic tumor surgery. Adv Tech Stand Neurosurg 22:343-373, 1995

36. Samii M, Matthies C: Management of 1000 vestibular schwannomas (acoustic neuromas): hearing function in 1000 tumor resections. Neurosurgery 40:248-262, 1997

37. Shelton C: Hearing preservation in acoustic tumor surgery. Otolaryngol Clin North Am 25:609-621, 1992

38. Spiegelmann R, Gofman J, Alezra D, et al: Radiosurgery for acoustic neurinomas (vestibular schwannomas). Isr Med Assoc J 1:8-13, 1999

39. Suh JH, Barnett GH, Sohn JW, et al: Results of linear accelerator-based stereotactic radiosurgery for recurrent and newly diagnosed acoustic neuromas. Int J Cancer 90:145-151, 2000

Manuscript received March 17, 2003.

Accepted in final form April 14, 2003.

Address reprint requests to: Georg Norén, M.D., Ph.D., New England Gamma Knife Center, Rhode Island Hospital, 593 Eddy Street, Providence, Rhode Island 02903. email georg_noren@ brown.edu. 